\title{
MINIMAL SURFACES WITH THE RICCI CONDITION IN 4-DIMENSIONAL SPACE FORMS
}

\author{
MAKOTO SAKAKI \\ (Communicated by Peter Li) \\ Dedicated to Professor Y. Hatakeyama on his 60 th birthday
}

\begin{abstract}
Let $X^{N}(c)$ denote the $N$-dimensional simply connected space form of constant curvature $c$. We consider a problem to classify those minimal surfaces in $X^{N}(c)$ which are locally isometric to minimal surfaces in $X^{3}(c)$. In this paper we solve this problem in the case where $N=4$, and give a result also in higher codimensional cases.
\end{abstract}

\section{INTRODUCTION}

Let $X^{N}(c)$ denote the $N$-dimensional simply connected space form of constant curvature $c$, and let $M$ be a minimal surface in $X^{N}(c)$ with Gaussian curvature $K(\leq c)$ with respect to the induced metric $d s^{2}$. When $N=3, M$ satisfies the Ricci condition with respect to $c$, that is, the metric $d \hat{s}^{2}=\sqrt{c-K} d s^{2}$ is flat at points where $K<c$. Conversely, every 2-dimensional Riemannian manifold with Gaussian curvature less than $c$ which satisfies the Ricci condition with respect to $c$, can be realized locally as a minimal surface in $X^{3}(c)$ (see [10]). Then it is an interesting problem to classify those minimal surfaces in $X^{N}(c)$ which satisfy the Ricci condition with respect to $c$, that is, to classify those minimal surfaces in $X^{N}(c)$ which are locally isometric to minimal surfaces in $X^{3}(c)$, or to compare locally the Riemannian structures of minimal surfaces in $X^{N}(c)$ with those of minimal surfaces in $X^{3}(c)$. In the case where $c=0$, Lawson [11] solved this problem completely (cf. Chapter IV of [12]). In [13], with some global assumptions, Naka (= Miyaoka) obtained some results in the case where $c>0$. In [14] we discussed exceptional minimal surfaces in $X^{N}(c)$ which satisfy the Ricci condition with respect to $c$.

The main purpose of this paper is to solve the above problem in the case where $N=4$.

Theorem 1. Let $M$ be a minimal surface in $X^{4}(c)$ with Gaussian curvature $K$ with respect to the induced metric $d s^{2}$. Suppose that the metric $d \hat{s}^{2}=$ $\sqrt{c-K} d s^{2}$ is flat at points where $K<c$. Then $M$ lies in a totally geodesic $X^{3}(c)$.

Received by the editors October 1, 1992.

1991 Mathematics Subject Classification. Primary 53A10. 
Remark 1. (i) When $c=0$, Theorem 1 is included in [11].

(ii) For $c>0$, there are flat minimal surfaces in $X^{5}(c)$ not lying in any totally geodesic $X^{4}(c)$, which automatically satisfy the Ricci condition with respect to $c$ (see $[2,8]$ ). So, in the case where $c>0$, Theorem 1 is not true if we replace $X^{4}(c)$ by $X^{5}(c)$.

We cannot apply the method used to prove Theorem 1 to higher codimensional cases directly. However in $\S 3$, with an additional assumption, we will give a result in higher codimensional cases.

\section{Preliminaries}

Let $M$ be a 2-dimensional Riemannian manifold isometrically immersed in $X^{N}(c)$ with Gaussian curvature $K$ with respect to the induced metric. Let $A$ be the second fundamental form of $M$. We denote by $T_{p} M$ and $T_{p}^{\perp} M$ the tangent space and the normal space of $M$ at $p$, respectively. A point $p$ on $M$ is called isotropic if the ellipse of curvature $\left\{A(X, X) \in T_{p}^{\perp} M ; X \in T_{p} M\right.$, $|X|=1\}$ at $p$ is a circle. We say that $M$ is isotropic if each point on $M$ is isotropic.

At each point $p$ on $M$, we choose orthonormal bases $\left\{e_{1}, e_{2}\right\}$ and $\left\{e_{3}, \ldots\right.$, $\left.e_{N}\right\}$ for $T_{p} M$ and $T_{p}^{\perp} M$, respectively. We shall make use of the following convention on the ranges of indices: $1 \leq i, j, k \leq 2,3 \leq \alpha, \beta \leq N$. Let $h_{i j}^{\alpha}$ be the components of $A$. We denote by $R_{\beta i j}^{\alpha}$ the components of the normal curvature tensor of $M$. Then

$$
R_{\beta i j}^{\alpha}=\sum_{k}\left(h_{i k}^{\alpha} h_{j k}^{\beta}-h_{j k}^{\alpha} h_{i k}^{\beta}\right) .
$$

Following [5], we define the normal scalar curvature $K_{n}$ of $M$ by

$$
K_{n}=\sum_{i<j, \alpha<\beta}\left(R_{\beta i j}^{\alpha}\right)^{2}=\frac{1}{4} \sum_{i, j, \alpha, \beta}\left(R_{\beta i j}^{\alpha}\right)^{2} .
$$

When $N=4$, we define the normal curvature $K_{\nu}$ of $M$ by $K_{\nu}=R_{412}^{3}$, which changes sign according to the orientation of the bases.

Assume further that $M$ is a minimal surface. Then we may choose the bases $\left\{e_{i}\right\}$ and $\left\{e_{\alpha}\right\}$ so that the components $h_{i j}^{\alpha}$ satisfy

$$
\left(h_{i j}^{3}\right)=\left(\begin{array}{cc}
\lambda & 0 \\
0 & -\lambda
\end{array}\right), \quad\left(h_{i j}^{4}\right)=\left(\begin{array}{cc}
0 & \mu \\
\mu & 0
\end{array}\right), \quad\left(h_{i j}^{\alpha}\right)=(0) \quad \text { for } \alpha \geq 5,
$$

for some $\lambda$ and $\mu$. It is easy to see that $(c-K)^{2}-K_{n} \geq 0$, and the point $p$ is isotropic if and only if $|\lambda|=|\mu|$, which is equivalent to that $(c-K)^{2}-K_{n}=0$. Similarly, when $N=4, c-K \geq\left|K_{\nu}\right|$, and $p$ is isotropic if and only if $c-K=\left|K_{\nu}\right|$.

\section{Proof OF THEOREM 1}

Proof of Theorem 1. Assume that $M$ lies fully in $X^{4}(c)$, namely, does not lie in a totally geodesic $X^{3}(c)$. Then $K<c$ and $K_{\nu} \neq 0$ open densely, where $K_{\nu}$ denotes the normal curvature of $M$ (see Lemma 2 of [7]). As the metric $d \hat{s}^{2}=\sqrt{c-K} d s^{2}$ is flat at points where $K<c$, we have

$$
\frac{K}{\sqrt{c-K}}-\frac{1}{2 \sqrt{c-K}} \Delta \log \sqrt{c-K}=0
$$


or equivalently,

$$
\Delta \log (c-K)=4 K
$$

at points where $K<c$, where $\Delta$ denotes the Laplacian of $M$ with respect to $d s^{2}$.

If $M$ is isotropic, then noting that an isotropic minimal surface lying fully in $X^{4}(c)$ is exceptional in the sense of [6], we have by Theorem $\mathrm{A}$ of [6],

$$
\Delta \log (c-K)=6 K-2 c
$$

at points where $K<c$. From (1) and (2) we have a contradiction. So $M$ is not isotropic.

Set

$$
M_{1}=\left\{p \in M ; K<c, K_{\nu} \neq 0, p \text { is not isotropic }\right\},
$$

which is an open dense subset of $M$. By Theorem 1 of [4],

$$
\Delta \log \left(c-K+K_{\nu}\right)=2\left(2 K-K_{\nu}\right)
$$

and

$$
\Delta \log \left(c-K-K_{\nu}\right)=2\left(2 K+K_{\nu}\right)
$$

on $M_{1}$. Set $F=K_{\nu} /(c-K)$. Then by (1), (3), and (4),

$$
\Delta F=-2(c-K) F\left(1+F^{2}\right)
$$

and

$$
|\nabla F|^{2}=2(c-K) F^{2}\left(1-F^{2}\right)
$$

on $M_{1}$, where $\nabla$ is the Riemannian connection of $M$ with respect to $d s^{2}$. We denote by $\tilde{K}, \tilde{\nabla}$, and $\tilde{\Delta}$ the Gaussian curvature, the Riemannian connection, and the Laplacian of $M_{1}$ with respect to the metric $d \tilde{s}^{2}=(c-K) d s^{2}$, respectively. We note that the metric $d \tilde{s}^{2}$ is nondegenerate on $M_{1}$. Then

$$
\tilde{K}=\frac{K}{c-K}-\frac{1}{2(c-K)} \Delta \log (c-K)=\frac{K}{K-c}
$$

on $M_{1}$, where we use (1) for the second equality. Equations (5) and (6) are rewritten as follows:

$$
\tilde{\Delta} F=-2 F\left(1+F^{2}\right)=: P(F)
$$

and

$$
|\tilde{\nabla} F|^{2}=2 F^{2}\left(1-F^{2}\right)=: Q(F)
$$

on $M_{1}$. As $0<|F|<1$ on $M_{1}, F$ is not constant on $M_{1}$ by (9). Hence we have

$$
Q \tilde{K}+\left(P-Q^{\prime}\right)\left(P-\frac{1}{2} Q^{\prime}\right)+Q\left(P^{\prime}-\frac{1}{2} Q^{\prime \prime}\right)=0
$$

on $M_{1}$, where the prime denotes the differentiation with respect to $F$ (see [3, p. $164 ; 9$, p. 136]). Noting that $0<|F|<1$ on $M_{1}$, we have by (7)-(10), $K=8 c / 9$ on $M_{1}$ and, by continuity, on $M$. As $K<c$ on $M_{1}$, we find that $c>0$. Now we have a contradiction because there are no minimal surfaces with constant curvature $8 c / 9$ in $X^{4}(c)$, where $c>0$ (see $[2,9]$ ).

Therefore, $M$ lies in a totally geodesic $X^{3}(c)$. 


\section{Higher CODIMENSIONAL CASES}

In this section we prove the following.

Theorem 2. Let $M$ be a minimal surface in $X^{N}(c)$ with Gaussian curvature $K$ with respect to the induced metric $d s^{2}$. Suppose that the metric $d \hat{s}^{2}=$ $\sqrt{c-K} d s^{2}$ is flat at points where $K<c$ and the normal scalar curvature of $M$ is constant. Then either (i) $M$ lies in a totally geodesic $X^{3}(c)$, or (ii) $c>0$ and $M$ is flat.

Remark 2. (i) A minimal surface $M$ in $X^{N}(c)$ lies in a totally geodesic $X^{3}(c)$ if and only if the normal scalar curvature of $M$ is identically zero (see Lemma 2 of [7]).

(ii) Flat minimal surfaces in $X^{N}(c)$ where $c>0$ are classified (see [2]). By Theorem 3.1(2) of [2] and Proposition 1(iii) of [8], we find that all of them have constant normal scalar curvature.

(iii) Minimal surfaces with constant normal scalar curvature in space forms are studied in [1] and [5].

Proof of Theorem 2. Assume that $M$ does not lie in a totally geodesic $X^{3}(c)$. Then $K<c$ open densely and the normal scalar curvature $K_{n}$ of $M$ is a positive constant (see Remark 2(i)).

When $M$ is isotropic, we have $(c-K)^{2}-K_{n}=0$ on $M$, and $K$ is a constant less than $c$. From the hypothesis that the metric $d \hat{s}^{2}=\sqrt{c-K} d s^{2}$ is flat at points where $K<c$, we have $c>0$ and $K=0$ on $M$.

When $M$ is not isotropic, set $M_{2}=\{p \in M ; K<c, p$ is not isotropic $\}$, which is an open dense subset of $M$. As the metric $d \hat{s}^{2}=\sqrt{c-K} d s^{2}$ is flat at points where $K<c$, we have

$$
\Delta \log (c-K)=4 K
$$

on $M_{2}$, where $\Delta$ denotes the Laplacian of $M$ with respect to $d s^{2}$. The argument to get (2.12) of [5] is valid on minimal surfaces in $X^{N}(c)$ except at isotropic points. Hence we have by (2.12) of [5] under our notation,

$$
\Delta \log \left\{(c-K)^{2}-K_{n}\right\}=8 K
$$

on $M_{2}$. By (11) and (12),

$$
\Delta K=6 K^{2}-6 c K-2 K_{n}-2 c K_{n} /(K-c)=: R(K)
$$

and

$$
|\nabla K|^{2}=2 K^{3}-4 c K^{2}+2\left(c^{2}-K_{n}\right) K=: S(K)
$$

on $M_{2}$, where $\nabla$ is the Riemannian connection of $M$ with respect to $d s^{2}$. If $K$ is not constant on $M_{2}$, then

$$
S K+\left(R-S^{\prime}\right)\left(R-\frac{1}{2} S^{\prime}\right)+S\left(R^{\prime}-\frac{1}{2} S^{\prime \prime}\right)=0
$$

on $M_{2}$, where the prime denotes the differentiation with respect to $K$ (see $[3$, p. $164 ; 9$, p. 136]). By the computation we find that (13) is a nontrivial equation for $K$. So $K$ must be constant on $M_{2}$, which is a contradiction. Hence $K$ is a constant less than $c$ on $M_{2}$, and by continuity, on $M$. From the hypothesis that the metric $d \hat{s}^{2}=\sqrt{c-K} d s^{2}$ is flat at points where $K<c$, we have $c>0$ and $K=0$ on $M$.

Therefore, either (i) $M$ lies in a totally geodesic $X^{3}(c)$ or (ii) $c>0$ and $M$ is flat. 


\section{REFERENCES}

1 A. C. Asperti, Minimal surfaces with constant normal curvature, J. Math. Soc. Japan 36 (1984), 375-386.

2. R. Bryant, Minimal surfaces of constant curvature in $S^{n}$, Trans. Amer. Math. Soc. 290 (1985), 259-271.

3. L. P. Eisenhart, An introduction to differential geometry, Princeton Univ. Press, Princeton, NJ, 1947.

4. I. V. Guadalupe and R. A. Tribuzy, Minimal immersions of surfaces into 4-dimensional space forms, Rend. Sem. Mat. Univ. Padova 73 (1985), 1-13.

5. T. Itoh, Minimal surfaces in a Riemannian manifold of constant curvature, Kodai Math. Sem. Rep. 25 (1973), 202-214.

6. G. D. Johnson, An intrinsic characterization of a class of minimal surfaces in constant curvature manifolds, Pacific J. Math. 149 (1991), 113-125.

7. K. Kenmotsu, On compact minimal surfaces with non-negative Gaussian curvature in a space of constant curvature. I, Tohoku Math. J. 25 (1973), 469-479.

8. __ On minimal immersions of $R^{2}$ into $S^{N}$, J. Math. Soc. Japan 28 (1976), 182-191.

9. __ Minimal surfaces with constant curvature in 4-dimensional space forms, Proc. Amer. Math. Soc. 89 (1983), 133-138.

10. H. B. Lawson, Complete minimal surfaces in $S^{3}$, Ann. of Math. (2) 92 (1970), 335-374.

11. _ Some intrinsic characterizations of minimal surfaces, J. Analyse Math. 24 (1971), 151-161.

12. __ Lectures on minimal submanifolds, Publish or Perish, Berkeley, 1980.

13. R. Naka, Some results on minimal surfaces with the Ricci condition, Minimal Submanifolds and Geodesics (M. Obata, ed.), Kaigai, Tokyo, 1978, pp. 121-142.

14. M. Sakaki, Exceptional minimal surfaces with the Ricci condition, Tsukuba J. Math. 16 (1992), 161-167.

Department of Mathematics, Faculty of Science, Hirosaki University, Hircsaki 036, JAPAN 